\title{
Reimplante dentário após avulsão traumática: 10 anos de acompanhamento
}

Tooth replantation after traumatic avulsion: 10 years of follow-up

Reimplantación dentaria tras avulsión traumática: 10 años de seguimiento

Luiz Eduardo de SOUZA 1

Izabela Volpato MARQUES 2

Alfredo Franco QUEIROZ3

Margareth Calvo Pessuti NUNES ${ }^{4}$

Nair Narumi Orita PAVAN ${ }^{5}$

Marcos Sérgio ENDO ${ }^{5}$

${ }^{1}$ Curso de Graduação em Odontologia do Departamento de Odontologia (DOD), Centro de Ciências da Saúde (CCS), Universidade Estadual de Maringá (UEM) 87083-170 Maringá - PR, Brasil

${ }^{2}$ Residência em Endodontia - Departamento de Odontologia (DOD), Centro de Ciências da Saúde (CCS), Universidade Estadual de Maringá (UEM) 87083-170 Maringá-PR, Brasil

3Professor Assistente em Endodontia - Departamento de Odontologia (DOD), Centro de Ciências da Saúde (CCS), Universidade Estadual de Maringá (UEM) 87083-170 Maringá-PR, Brasil

${ }^{4}$ Professora Adjunta em Dentística - Departamento de Odontologia (DOD), Centro de Ciências da Saúde (CCS), Universidade Estadual de Maringá (UEM) 87083-170 Maringá-PR, Brasil

${ }^{5}$ Professor(a) Adjunto em Endodontia - Departamento de Odontologia (DOD), Centro de Ciências da Saúde (CCS), Universidade Estadual de Maringá (UEM) 87083-170 Maringá -PR, Brasil

\section{Resumo}

A avulsão dentária consiste em um deslocamento completo do dente para fora do alvéolo e o seu reimplante imediato é a opção mais favorável, porém sob algumas condições nem sempre é possível. O objetivo deste estudo é relatar um caso em que houve o reimplante dentário tardio e um acompanhamento ao longo de 10 anos, não apresentando reabsorção radicular. Paciente do sexo masculino, 6 anos de idade, sofreu avulsão do dente 21 causada por queda de bicicleta. O dente foi colocado em um copo com soro fisiológico e reimplantado no hospital em duas horas, sendo instalada uma esplintagem não-rígida e após 11 dias removida. Ao exame radiográfico, foi observado que o dente apresentava-se com rizogênese incompleta, com paredes finas e o ápice aberto (estágio 9 de Nolla). Clinicamente, um mês após o trauma, o dente apresentou-se assintomático e sem mobilidade, com resposta inconclusiva ao teste de sensibilidade pulpar ao frio. Com a interrupção do desenvolvimento radicular e a presença de rarefação óssea periapical, realizou-se o teste de cavidade pulpar e foi planejado o tratamento com posterior intervenção endodôntica. Dez anos após o trauma dentário, o dente apresentava-se sem mobilidade, com resposta negativa ao teste de palpação e percussão vertical. A radiografia mostrou-se com aspectos de normalidade, não havendo presença de reabsorção dentária. Conclui-se que o reimplante dentário apresentou um prognóstico favorável, não exibindo reabsorção radicular inflamatória ou por substituição, além de restabelecer estética e função durante 10 anos.

Descritores: Avulsão Dentária; Reimplante Dentário; Reabsorção de Dente; Traumatismos Dentários.

\section{Abstract}

Dental avulsion consists of a complete displacement of a tooth out of the alveolar bone. Its immediate replantation is the most favorable option; however, under some conditions, it is not always possible. This study aimed at reporting a case treated by means of late tooth replantation whose follow-up was monitored over 10 years with no root resorption. A 6-year-old male patient had suffered avulsion of tooth 21 when falling down from a bicycle. The tooth had been laid in a glass with saline solution and replanted in the hospital after two hours by placing a non-rigid splint and removed after 11 days. The radiographic testing revealed that the tooth had an incomplete rhizogenesis, with thin walls and the apex open (Nolla's 9 stage). Clinically, one month after the trauma, the tooth was asymptomatic and without mobility, with an inconclusive response to the pulp sensitivity testing to cold. Considering the interruption of the root development and the presence of periapical bone rarefaction, the pulp cavity testing was carried out and the treatment was planned with a later endodontic intervention. Ten years after dental trauma, the tooth showed no mobility, with a negative response to the palpation and vertical percussion tests. The radiography showed the tooth with a normal aspect, and with no root resorption. It was concluded that the tooth replantation had a favorable prognosis, showing no inflammatory reaction either due to root resorption or replacement, in addition to restored aesthetics and function for 10 years.

Descriptors: Tooth Avulsion; Tooth Replantation; Tooth Resorption; Tooth Injuries.

\section{Resumen}

La avulsión dental consiste en un desalojo completo del diente para fuera del alvéolo y su reimplante inmediato es la opción más favorable, pero, bajo algunas condiciones, no siempre es posible. El objetivo de este estudio es relatar un caso en el que hubo el reimplante dentario tardío y un acompañamiento a lo largo de 10 años, no presentando reabsorción radicular. Paciente del sexo masculino, 6 años de edad, sufrió avulsión del diente 21 causada por caída de bicicleta. El diente fue colocado en un vaso con solución salina, reimplantado en el hospital en dos horas, siendo instalada un esplintaje no-rígida y 11 días fue quitado. En el examen radiográfico, fue observado que el diente se presentaba con rizogénesis incompleta, con paredes finas y el ápice abierto (etapa 9 de Nolla). Clínicamente, un mes después del trauma, el diente se presentó asintomático y sin movilidad, con respuesta no concluyente al test de sensibilidad pulpar al frío. Con la interrupción del desarrollo radicular y la presencia de rarefacción ósea periapical, se realizó el test de la cavidad pulpar y fue planificado el tratamiento con posterior intervención endodóntica. Diez años después del trauma dentario, el diente se presentaba sin movilidad, con respuesta negativa al test de palpación y percusión vertical. La radiografía se mostró con aspectos de normalidad, no habiendo presencia de reabsorción dentaria. Se concluye que el reimplante dentario presentó un pronóstico favorable, no exhibiendo reabsorción radicular inflamatoria o por sustitución, además de restablecer la estética y función durante 10 años.

Descriptores: Avulsión de Diente; Reimplante Dental; Resorción Dentaria; Traumatismos de los Dientes.

INTRODUÇÃO

A avulsão dentária é frequentemente causada por um trauma severo ${ }^{1}$ e consiste em um deslocamento completo do dente para fora do alvéolo ${ }^{2,3}$, resultando na ruptura do epitélio gengival, do ligamento periodontal, prejuízos ao cemento, ao osso alveolar e rompimento do feixe vasculonervoso ${ }^{4-6}$.

Ocorre com maior frequência nos incisivos superiores, sendo o sexo masculino de maior prevalência ${ }^{7}$. Um dos fatores importantes 
no prognóstico é o tempo extra-alveolar ${ }^{8}$. O reimplante imediato é a opção mais favorável diante à avulsão, no entanto sob algumas condições nem sempre é possível ${ }^{9}$. Neste caso, é necessário um meio de armazenamento que possa preservar a viabilidade das células do ligamento periodontal ${ }^{10,11}$. Danos severos ao ligamento periodontal poderão ocasionar a anquilose e a reabsorção por substituição, enquanto que na presença de infecção pulpar causará a reabsorção radicular inflamatória ${ }^{12}$. Quando danos irreversíveis aos cementoblastos e outras células do ligamento periodontal ocorrem, há uma reação inflamatória que estimula a ação de clastos, com reabsorção de cemento e dentina radicular ${ }^{13}$.

A prevalência de desfechos desfavoráveis de reimplante dentário é de $57 \%$ a $80 \%{ }^{13}$, uma vez que alguns dos dentes reimplantados têm menores chances de sobrevivência a longo prazo e podem até mesmo ser perdidos ou extraídos em um estágio posterior ${ }^{14-16}$.

O objetivo deste estudo é relatar um caso em que houve o reimplante dentário e um acompanhamento ao longo de 10 anos, não apresentando reabsorção radicular.

\section{CASO CLÍNICO}

Paciente do sexo masculino, 6 anos de idade, compareceu ao Centro Especializado Maringaense de Traumatismo em Odontologia (CEMTrau-Odonto) devido a avulsão do incisivo central esquerdo (21), causada por queda de bicicleta. O responsável relatou durante a anamnese que após a avulsão o dente foi imediatamente colocado em um copo com soro fisiológico, levado ao hospital e reimplantado em duas horas, sendo instalada uma esplintagem do tipo não-rígida, a qual após 11 dias foi removida. Paciente encontrava-se com vacina antitetânica em dia. Ao exame radiográfico, observou-se que o dente apresentava-se com rizogênese incompleta, com paredes finas e o ápice ainda aberto (estágio 9 de Nolla) (Figura 1). Clinicamente, um mês após o trauma, o dente apresentou-se assintomático e sem mobilidade, com resposta inconclusiva ao teste de sensibilidade pulpar ao frio (Maquira, Maringá/PR, Brasil), negativo à palpação e a percussão vertical.

Com a interrupção do desenvolvimento radicular e a presença de rarefação óssea periapical (Figura 2), realizou-se o teste de cavidade pulpar, planejou-se o tratamento e iniciou-se a intervenção endodôntica. Realizouse a abertura coronária com ponta diamantada esférica \#1012 (KG Sorensen, Cotia/SP, Brasil), forma de contorno e conveniência com ponta diamantada tronco-cônica de extremidade inativa \#3082 (KG Sorensen, Cotia/SP, Brasil).

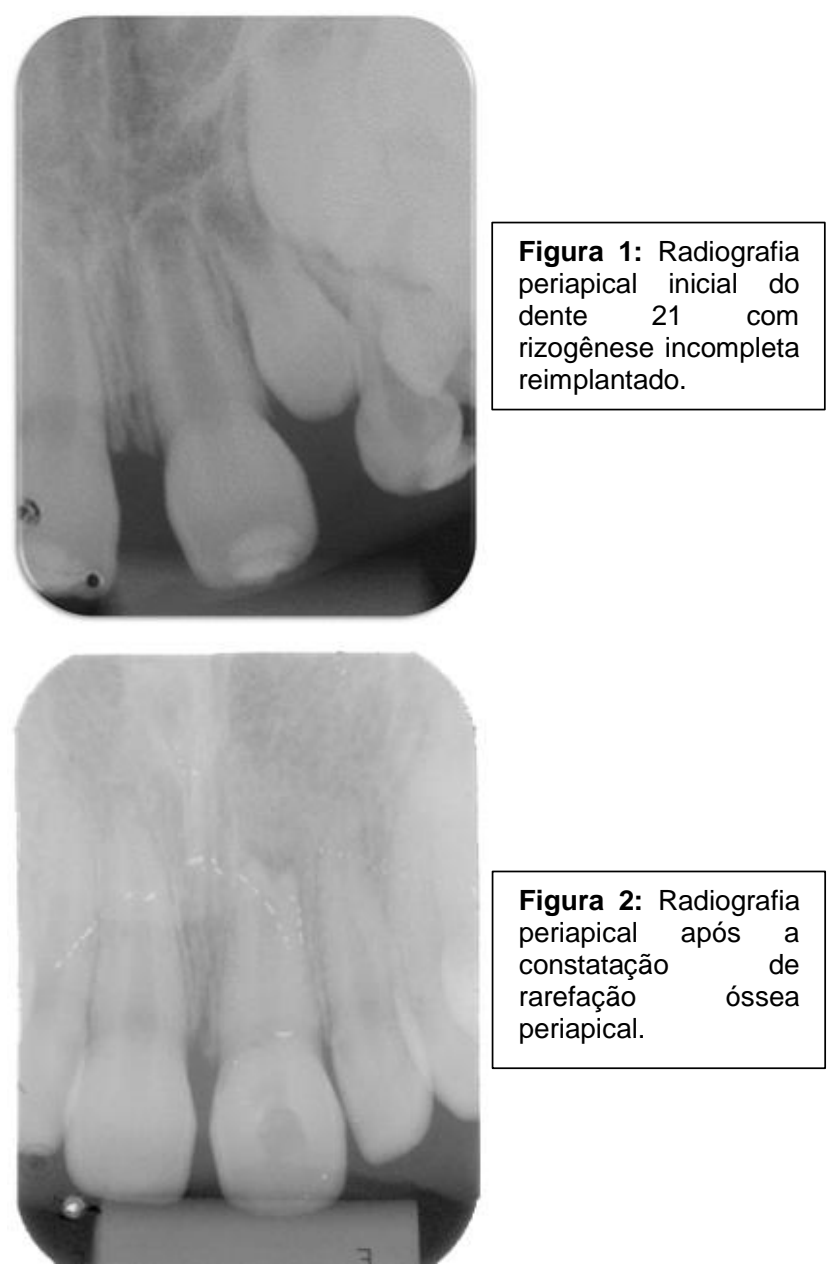

A partir da odontometria radiográfica, foi realizada a instrumentação manual e irrigação com hipoclorito de sódio (NaOCl) 1\% (Indústria Química Ltda, São Caetano do Sul/SP, Brasil). Em seguida efetuou-se a irrigação com EDTA 17\% (Biodinâmica, Ibiporã/PR, Brasil) mantendo em posição durante 3 minutos. Após irrigação com $\mathrm{NaOCl} 1 \%$ e secagem do canal radicular com cones de papel absorvente (Dentsply Sirona, Pirassununga/SP, Brasil), inseriu-se a medicação intracanal à base de hidróxido de cálcio P.A. (Biodinâmica, Ibiporã/PR, Brasil) associado ao veículo propilenoglicol. Após 3 trocas de medicação, aproximadamente a cada 3,6 meses, notou-se a formação de uma barreira mineralizada na região apical, possibilitando ser realizada a obturação do canal através da técnica do cone moldado \#80 e condensação lateral com cimento endodôntico a base de óxido de zinco e eugenol, selamento duplo com guta-percha em bastão, restauração provisória com IRM (Rio de Janeiro/RJ, Brasil) (Figura 3), sendo na semana seguinte restaurado definitivamente com resina composta. Os controles clínicos e radiográficos 
foram realizados anualmente durante os 6 primeiros anos após o trauma, sendo posteriormente realizados a cada 2 anos por mais 4 anos. Clinicamente, 10 anos seguidos do trauma dentário, o dente apresentava-se sem mobilidade, com resposta negativa ao teste de palpação e percussão vertical (Figura 4). 0 exame radiográfico mostrou-se com aspectos de normalidade, não havendo reabsorção dentária (Figura 5).
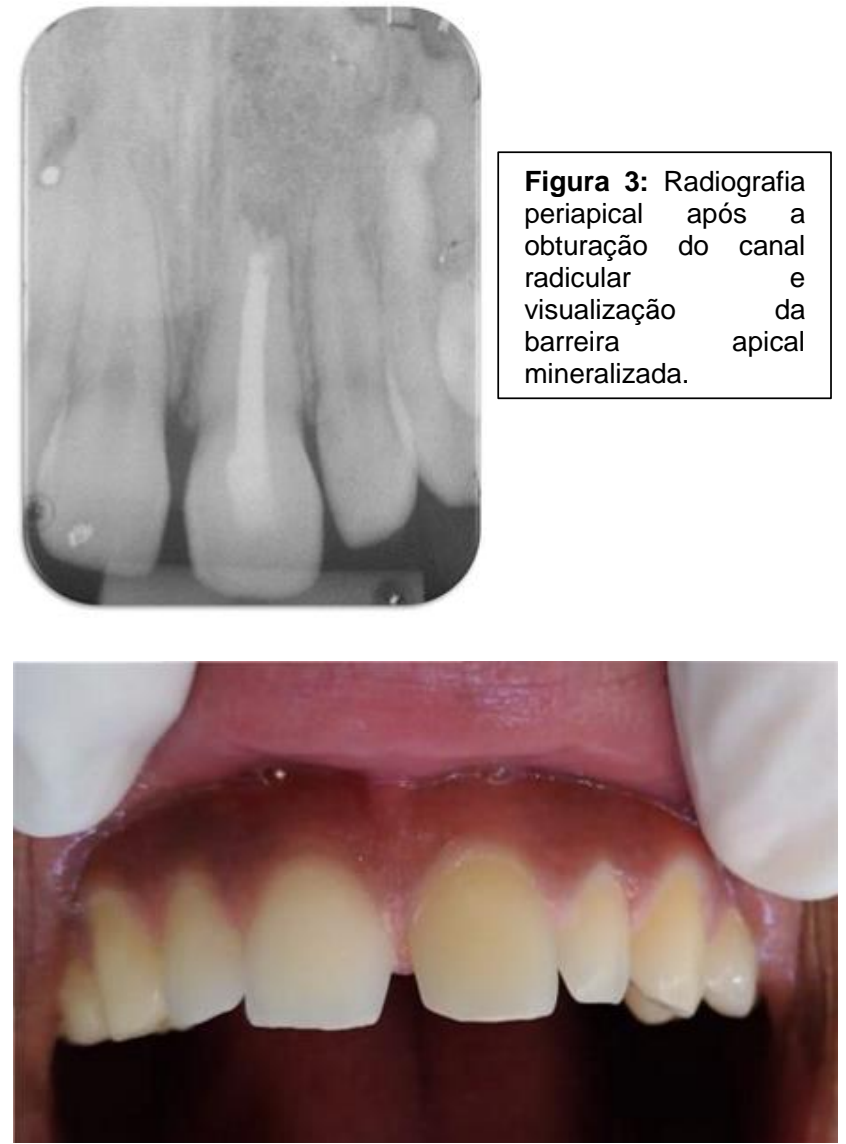

Figura 4: Avaliação clínica do dente 21 após 10 anos do trauma.

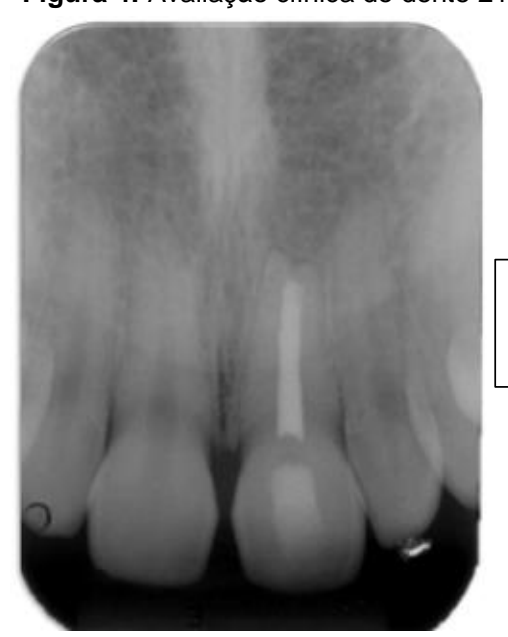

Figura 5: Radiografia periapical 10 anos após o trauma.

DISCUSSÃO

Após um período extra-alveolar de 60 minutos a seco, praticamente não é esperado viabilidade das células do ligamento periodontal ${ }^{17}$. O meio de armazenamento e o período extra-alveolar são fatores críticos para cura do ligamento periodontal de um dente avulsionado. As células do ligamento periodontal hidratadas manterão sua viabilidade, permitindo-lhes se conectar no reimplante causando uma inflamação destrutiva mínima ${ }^{18}$. Em um estudo in vivo, a reabsorção inflamatória foi comum após o armazenamento a seco e foi relacionada a duração do período extra-alveolar. No mesmo trabalho a reabsorção por substituição também exibiu uma forte relação com o armazenamento a seco e tornou-se proeminente após 60 minutos, no entanto, o trabalho concluiu que a solução salina e a saliva ofereceram proteção contra reabsorção radicular durante 0 período extra-alveolar ${ }^{19}$. Outro trabalho revelou que o armazenamento na solução salina após 30 minutos a seco não influencia no reparo periodontal e pulpar, por outro lado, parece ser aceitável no sentido de não infligir mais danos às estruturas ${ }^{20}$. A situação ideal é reimplantar 0 dente imediatamente após a avulsão, pois o tempo extra-oral é um fator determinante para o sucesso do tratamento e para um bom prognóstico $^{21}$. No entanto, nem sempre é possível, sendo necessário um meio de armazenamento que produza condições que assemelham-se ao do alvéolo, com osmolalidade adequada, $\mathrm{pH}$, metabólitos nutricionais e glicose, e assim criar as melhores condições possíveis para o armazenamento. A solução salina tem $\mathrm{pH}$ e osmolaridade fisiológica, porém, não contém íons essenciais e glicose que são fundamentais para a viabilidade das células periodontais ${ }^{22-24}$. No seguinte caso, o reimplante imediato não foi possível, sendo o dente armazenado na solução citada acima por um tempo de duas horas. Neste obteve-se uma resposta satisfatória, visto que o dente não apresentou qualquer tipo de reabsorção radicular. Embora a resposta tenha se apresentado favorável, nem sempre iremos nos deparar com essa situação, por não ser as condições ideais de tempo e armazenamento.

A esplintagem recomendada é do tipo não-rígida, devendo permitir o movimento fisiológico do dente durante a reparação periodontal ${ }^{25}$, por um período de tempo mínimo, 1-2 semanas, esperando uma menor incidência de reabsorção por substituição e anquilose ${ }^{26}$. Corroborando com a utilização da esplintagem com monofilamento de nylon, que mostrou-se favorável para a reparação após o reimplante dentário, mantida por um período de 11 dias. Além disso, a linha de nylon tem se apresentado de forma interessante, devido a sua flexibilidade, baixo custo e estética ${ }^{27}$.

A intervenção endodôntica em dentes 
com rizogênese completa é preconizada ser realizada de 7 a 14 dias após o reimplante. Quando realizada antes do reimplante aumenta a chance de reabsorção por substituição e a reabsorção superficial ${ }^{28}$. Para o dente com ápice aberto, o objetivo essencial é promover a revascularização da polpa, nesta ocasião, evitar a infecção pulpar é de suma importância ${ }^{29}$. Uma vantagem da revascularização é a possibilidade de um desenvolvimento radicular, reforçando as paredes dentinárias e fortalecendo a raiz contra a fratura $^{30}$, sendo 0 início do tratamento endodôntico evitado, se possível, a menos que sinais definidos de necrose pulpar, como a inflamação perirradicular, estejam presentes ${ }^{18}$. Em um estudo através de análise radiográfica mostrou que de 72 dentes imaturos (largura do forame apical 1,1-5,0 mm) apenas $13(18 \%)$ apresentaram revascularização pulpar ${ }^{31}$.

No caso de necrose pulpar e infecção em dente com o ápice aberto, é necessário a realização da descontaminação do canal e a estimulação da apicificação ${ }^{32,33}$. A proposta do tratamento é induzir a formação de uma barreira biológica calcificada através do forame apical, para permitir a condensação do material obturador no canal ${ }^{34}$, sendo a barreira formada a partir de trocas regulares da pasta de hidróxido de cálcio $\left(\mathrm{Ca}(\mathrm{OH})_{2}\right)$, que geralmente são renovadas a cada 3 meses $^{35}$. O tempo necessário para a formação de uma barreira apical na apicificação com $\mathrm{Ca}(\mathrm{OH}) 2$ pode ser considerável, geralmente com a duração de 20 meses e outras condições como idade e presença de sintomas ou radioluscências perirradiculares podem afetar 0 tempo necessário para formar uma barreira apical ${ }^{36}$.

A reparação apical e perirradicular dos dentes com rizogênese incompleta pode ser efetuada às custas de odontoblastos, da papila dentária e bainha de Hertwig, dos cementoblastos e células mesenquimais indiferenciadas e jovens do ligamento periodontal ${ }^{37}$. Além da formação de tecido mineralizado, a pasta possibilita a eliminação de forma eficiente das bactérias do interior do canal radicular através do contato direto ${ }^{38}$.

Neste caso, devido a presença do ápice aberto e a possibilidade de se estabelecer a revascularização pulpar, aliado ao teste de sensibilidade pulpar inconclusivo em paciente infantil, inicialmente foi optado por evitar o tratamento endodôntico e realizar os acompanhamentos por meio dos controles clínico-radiográficos. Uma vez constatado a necrose pulpar, após o teste de cavidade e a presença de uma rarefação óssea periapical, além de não exibir o desenvolvimento radicular, foi iniciado a intervenção, buscando-se através da apicificação a formação de uma barreira mineralizada, que foi notada aproximadamente um ano do tratamento após as trocas de medicação intracanal.

\section{REFERENCIAS}

1. Petrovic B, Markovic D, Peric T, Blagojevic D. Factors related to treatment and outcomes of avulsed teeth. Dent Traumatol. 2010;26(1):52-9.

2. Wang SH, Chung MP, Su WS, Cheng JC, Shieh YS. Continued root formation after replantation and root canal treatment in an avulsed immature permanent tooth: a case report. Dent Traumatol. 2010;26(2):182-85.

3. Zhan X, Zhang C, Dissanayaka WL, Cheung GSP, Jin L, Yang $Y$, et al. Storage media enhance osteoclastogenic potential of human periodontal ligament cells via RANKLindependent signaling. Dent Traumatol. 2012;29(1):59-65.

4. Andreasen JO, Andreasen FM. Avulsions. Textbook and color atlas of traumatic injuries to the teeth. St Louis: Mosby; 1994. p.383-425.

5. Barrett EJ, Kenny DJ. Avulsed permanent teeth: a review of the literature and treatment guidelines. Endod Dent Traumatol. 1997;13(4):153-63.

6. Andreasen JO, Andreasen FM. Essentials of traumatic injuries to the teeth. Copenhagen: Munksgaard Mosby; 2000. p.113-31.

7. Majorana A, Bardellini E, Conti G, Keller E, Pasini S. Root resorption in dental trauma: 45 cases followed for 5 years. Dent Traumatol. 2003;19(5):262-65.

8. Andersson L, Bodin I. Avulsed human teeth replanted within 15 minutes - a long-term clinical follow-up study. Dent Traumatol. 1990;6(1):37-42.

9. Flores MT, Andersson L, Andreasen JO, Bakland LK, Malmgren B, Barnett $F$, et al. Guidelines for the management of traumatic dental injuries. II. Avulsion of permanent teeth. Dent Traumatol. 2007;23(3):130-36.

10. Ozan F, Polat ZA, Er K, Ozan U, Deger O. Effect of Propolis on Survival of Periodontal Ligament Cells: New Storage Media for Avulsed Teeth. J Endod. 2007;33(5):570-73.

11. Bag I, Yildirim S. Effect of avulsion storage media on periodontal ligament fibroblast differentiation. Dent Traumatol. 2017; 33(6):458-64.

12. Pohl $Y$, Filippi A, Kirschner $H$. Results after replantation of avulsed permanent teeth. I. Endodontic considerations. Dent Traumatol. 2005;21(2):80-92.

13. Roskamp L, Silva Neto UX, Carneiro E, Fariniuk LF, Westphalen VPD. Influence of Atopy in the Outcome of Avulsed and Replanted Teeth during 5 Years of Follow-up. J Endod. 2017;43(1):25-8. 
14. Andreasen JO, Borum MK, Jaeobsen HE, Andreasen FM. Replantation of 400 avulsed permanent incisors. 1. Diagnosis of healing complications. Dent Traumatol. 1995;11(2): 51-8.

15. Hecova H, Tzigkounakis V, Merglova V, Netolicky J. A retrospective study of 889 injured permanent teeth. Dent Traumatol. 2010; 26(6):466-75.

16. Andersson L, Andreasen JO, Day P, Heithersay G, Trope M, DiAngelis AJ, et al. International Association of Dental Traumatology guidelines for the management of traumatic dental injuries: 2. Avulsion of permanent teeth. Dent Traumatol. 2012;28(2):88-96.

17. Blomlof L, Andersson L, Lindskog S, Hedstrom K.-G, Hammarstrom L. Periodontal Healing of Replanted Monkey Teeth Prevented from Drying. Acta Odontol Scand. 1983;41(2): 117-23.

18. Trope M. Clinical Management of the avulsed tooth: present strategies and future directions. Dent Traumatol. 2002;18(1):1-11.

19. Andreasen JO. Effect of extra-alveolar period and storage media upon periodontal and pulpal healing after replantation of mature permanent incisors in monkeys. Int $\mathrm{J}$ Oral Surg. 1981; 10(1):43-53.

20. Andreasen JO, Schwartz O. The effect of saline storage before replantation upon dry damage of the periodontal ligament. Dent Traumatol. 1986; 2(2):67-70.

21. Poi W, Sonoda C, Martins C, Melo M, Pellizer E, et al. Storage Media For Avulsed Teeth: A Literature Review. Braz Dent J. 2013;24(5): 437-45.

22. Çaglar E, Sandalli N, Kuscu OO, Durhan MA, Pisiriciler $\mathrm{R}$, et al. Viability of fibroblasts in a novel probiotic storage media. Dent Traumatol. 2010;26(5):383-87.

23. Goswami M, Chaitra TR, Chaudhary S, Manuja $\mathrm{N}$, Sinha A. Strategies for periodontal ligament cell viability: an overview. J Conserv Dent. 2011;14(3):215-20.

24. Malhotra N. Current developments in interim transport (storage) media in dentistry: an update. Br Dent J. 2011;211(1):29-33.

25. von Arx T, Filippi A, Buser D. Splinting of traumatized teeth with a new device: TTS (Titanium Trauma Splint). Dent Traumatol. 2001;17(4):180-84.

26. Antrim DD, Ostrowski JS. A functional splint for traumatized teeth. J Endod. 1982;8(7):328-31.

27. Ben Hassan MW, Andersson L, Lucas PW. Stiffness characteristics of splints for fixation of traumatized teeth. Dent Traumatol. 2016;32(2):140-45.

28. Andreasen JO. The effect of pulp extirpation or root canal treatment on periodontal healing after replantation of permanent incisors in monkeys. J Endod. 1981;7(6):245-52.

29. Sahin S, Saygun NI, Kaya Y, Ozdemir A. Treatment of complex dentoalveolar injury avulsion and loss of periodontal tissue: a case report. Dent Traumatol. 2008;24(5):581-84.

30. Trope M. Treatment of immature teeth with nonvital pulps and apical periodontitis. Endod Topics. 2006;14:51-9.

31. Kling $M$, Cvek M, Mejare I. Rate and predictability of pulp revascularization in therapeutically reimplanted permanent incisors. Endod Dent Traumatol.1986;2(3):83-9.

32. Villa $P$, Fernández $R$. Apexification of a replanted tooth using mineral trioxide aggregate. Dent Traumatol. 2005;21(5):306-8.

33. Soares J, Santos S, César C, Silva P, Sá M, Silveira F. Calcium hydroxide induced apexification with apical root development: a clinical case report. Int Endod J. 2008;41(8):710-19.

34. Chosack A, Sela J, Cleaton-Jones P. A histological and quantitative histomorphometric study of apexification of nonvital permanent incisors of vervet monkeys after repeated root filling with a calcium hydroxide paste. Dent Traumatol. 1997;13(5):211-17.

35. Rafter M. Apexification: a review. Dent Traumatol. 2005;21(1):1-8.

36. Huang G. Apexification: the beginning of its end. Int Endod J. 2009;42(10):855-66.

37. Lopes HP, Siqueira Jr JF. Endodontia: biologia e técnica. Rio de Janeiro: Elsevier; 2015. p.772-3.

38. Bystrom A, Claesson R, Sundqvist G. The antibacterial effect of camphorated paramonochlorophenol, camphorated phenol and calcium hydroxide in the treatment of infected root canals. Endod Dent Traumatol. 1985;1(5):170-75.

\section{CONFLITO DE INTERESSES}

Os autores declaram não haver conflitos de interesse

\section{AUTOR PARA CORRESPONDÊNCIA}

\author{
Marcos Sérgio Endo \\ Departamento de Odontologia da Universidade \\ Estadual de Maringá - UEM \\ Av. Mandacaru 1550 \\ 87080-000 Maringá-PR, Brasil \\ E-mail: marcossendo@gmail.com
}

Submetido em 17/06/2019 Aceito em 23/10/2020 